\title{
The Application of NIR Process Analytical Chemistry in Studying Competitive Adsorption Process
}

\author{
Hong-Wei Yang ${ }^{1}$, Chen-Bo Cai ${ }^{1,}$, , Lu Xu ${ }^{2}$, Lun Li ${ }^{3}$, Yan-Li Zou ${ }^{1}$, Yong-Yuan Tao ${ }^{1}$, Mei-Qiong Wen ${ }^{1}$ \\ ${ }^{1}$ College of Chemistry and Life Science, Chuxiong Normal University, Chuxiong, PR China \\ ${ }^{2}$ College of Material and Chemical Engineering, Tongren University, Tongren, PR China \\ ${ }^{3}$ Department of Science and Technology, Chuxiong Normal University, Chuxiong, PR China
}

Email address:

ccp66516@163.com (Chen-Bo Cai)

${ }^{*}$ Corresponding author

\section{To cite this article:}

Hong-Wei Yang, Chen-Bo Cai, Lu Xu, Lun Li, Yan-Li Zou, Yong-Yuan Tao, Mei-Qiong Wen. The Application of NIR Process Analytical Chemistry in Studying Competitive Adsorption Process. American Journal of Applied Chemistry. Vol. 7, No. 3, 2019, pp. 80-86. doi: 10.11648/j.ajac.20190703.11

Received: April 8, 2019; Accepted: May 21, 2019; Published: June 12, 2019

\begin{abstract}
With an experimental setup of near-infrared process analytical chemistry, a competitive adsorption process of orthoxylene and aniline vapor onto silica gel has been revealed in the paper. The mixture vapor of orthoxylene and aniline has been introduced into a quartz adsorption bed, which was filled with adsorbent of silica gel and monitored continuously by a near-infrared spectrometer. Based on near-infrared spectra recorded during the adsorption process and chemometrics methodologies, the competitive adsorption process has been studied completely as well as clearly: 1) at about 62 minutes the adsorption achieved its equilibrium or stable state with aniline concentration of $0.22 \mathrm{~g} / \mathrm{g}$, and without any orthoxylene; 2) othoxylene was adsorbed first, but then rapidly replaced by aniline; 3 ) the adsorption of aniline resulted from the hydrogen bonds between aniline's amino groups and silica gel's silanol groups while that of orthoxylene was due to physical adsorption; 4) aniline was adsorbed vertically on the silica gel but orthoxylene laid evenly; 5) some surface of silica gel was more active for adsorption than others.
\end{abstract}

Keywords: Gas-Solid Competitive Adsorption, Near-Infrared Spectroscopy (NIR), Process Analytical Chemistry (PAC), Chemometrics, Orthoxylene, Aniline, Silica Gel

\section{Introduction}

From the standpoint of chemistry and chemical engineering, a complete understanding of a process of gas-solid adsorption process means: 1) thermodynamic of the process, such as its equilibrium concentration; 2) kinetics of the process, mainly, the adsorption rate of every adsorbate at any time; 3) the interaction between adsorbate and adsorbent, and 4) the adsorption state, namely, how the gas molecules are adsorbed on the surface of adsorbent [1-3]. Thermodynamic and kinetic of the process is indispensable to designing or operating industrial adsorption equipment, while the interaction between adsorbate adsorbent as well as the adsorption state are important for chemists to comprehend the mechanism of the adsorption process completely. A number of traditional methods have been employed to study the thermodynamic or kinetics of adsorption process for a long time [4-6]. However, as for information about the interaction or adsorption states, only few experimental methods are suitable. Among them diffuse reflectance infrared spectroscopy (IR) is the most commonly used because the alterations of some vibrations could be recorded in IR spectra after adsorbate molecules have been adsorbed by adsorbent [7-9].

Of course, researchers can employ different methods to detect different information described above, respectively. But obviously, a better way is to collect as much information as possible with the same method and at the same time. Besides more efficient, information recorded in this way is synchronous, making it possible to combine all information for understanding the mechanism of an adsorption process much more clearly and thoroughly.

Of infrared spectroscopy, near-infrared (NIR) process 
analytical chemistry (PAC) is more appropriate to satisfy the above demands as compared with mid-infrared, because it is convenient to trace an adsorption process continuously via an optical fiber probe during the whole adsorption process without disturbing the practical process, and simulate an industrial adsorption bed [10-12].

NIR PAC is very suitable for multi-component or competitive adsorptions that are more common than single-component adsorption in nature or industry, and also more difficult for traditional methods to study, since the amount of each adsorbent and their adsorption states are all preserved in the NIR spectra. Unlike the former references that focus on experimental set and data treatment of chemometrics [13, 14], in this paper, we have selected orthoxylene/aniline as adsorbates that have similar molecular structure, and silica gel as adsorbent that is widely used in industry, to demonstrate how the complicated competitive adsorption process could be revealed by NIR PAC in detail.

\section{Experimental}

\subsection{Materials}

Orthoxylene (AR); aniline (AR); tetrachloride carbon (AR); silica gel (Technical grade, Qingdao Haiyang Chemical Co., $\mathrm{Ltd})$

\subsection{Experimental Setup and Measurement}

The experimental setup is demonstrated in Figure 1: a small quartz rectangle cell that has been filled with silica granules and monitored by a spectrometer probe, and the gas introduced is the vapor mixture composed of orthoxylene, aniline and nitrogen gas. It is worth noting that what the spectrometer probe monitored is granules of silica gel rather than the gaseous phase. At each one minute, the NIR spectrometer (Nicolet Nexus 870) records a spectrum during the adsorption process until adsorption equilibrium state.

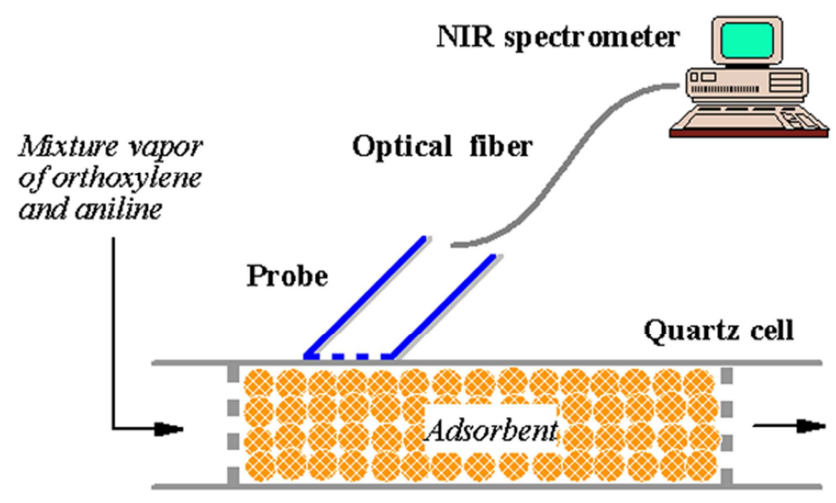

Figure 1. The experimental setup.

Unlike transmission spectroscopy, the strength of diffuse reflectance spectroscopy is usually recorded in terms of reflectance ratio $(\mathrm{R})$, for $\lg (1 / \mathrm{R})$ may roughly have linear relationship with concentration of a certain analyte.

\subsection{Preparation of Calibration Samples and Validation Samples}

It is necessary for NIR analysis to have a calibration (or training) set for building calibration models, as well as also a validation (or test) set for evaluating the predication error of the calibration models. For increasing the predication capacity of the quantitative calibration model, in the paper calibration as well as validation samples are prepared also in the above experimental setup. High performance liquid chromatography (HPLC) has been employed to determine their actual concentrations. The concentration of orthorxylene or aniline upon the silica gel is represented as the ratio of the adsorbate to silica gel by weight $(\mathrm{g} / \mathrm{g})$.

\section{Chemometrics Strategy and Algorithm}

\subsection{Signal Separation and Variable Selection}

Unlike a single-component adsorption, for a multi-component system raw NIR spectra usually should be preprocessed by signal separation in order to discriminate which component results in a certain absorbance peak, and select wavebands (variables) for constructing quantitative calibration models. Of course, it's easy to assign some spectral peaks to a certain group, such as the peak around $6568 \mathrm{~cm}^{-1}$ to the first overtone of $\mathrm{N}-\mathrm{H}$ stretching vibration of amino in aniline adsorbed upon silica gel surface, but there are most wavebands in which spectroscopic signal are overlapped, for example, the waveband between $4350-4000 \mathrm{~cm}^{-1}$, in which both orthoxylene and aniline have NIR signals due to vibrations of alkyl. For these overlapped wavebands, it is necessary to separate their firstly in order to select more appropriate variables for increasing the predication capacity of quantitative calibration models constructed afterwards.

Over the last decades, wavelet analysis has been becoming a frequently-used and powerful algorithm to separate complex signals, and for NIR spectra there also have been a number of works $[15,16]$. Most works of wavelet analysis are based upon the decomposition coefficients, but in the paper, it is based upon wavelet reconstruction, since the reconstructed NIR spectra are more straightforward for assignment of some spectral peaks.

After decomposing and then reconstructing the raw NIR spectra at various decomposition levels, some wavebands could be discovered, at which the strengths of reconstructed signals of one component are much larger than another component. These wavebands are characteristic wavebands, and therefore should be selected as variables of the component to construct its quantitative calibration model.

\subsection{Construction of Quantitative Calibration Models}

There have been a variety of mature algorithms to build linear quantitative calibration models in chemometrics, like principle component regression (PCR) or partial least squares (PLS) regression $[15,17,18]$. In our case, from the beginning of the adsorption process to the adsorption equilibrium the 
concentration of orthoxylene and aniline may alter greatly (from $0 \%$ to more than $20 \%$ ), making nonlinear problem not insignificant. Therefore, in the paper back propagation-artificial neural network (BP-ANN) has been used to solve the problem [18, 19]. PLS has also been employed, but not for building quantitative calibration models, instead for compressing spectral data as well as reducing colinearity as a pre-treatment algorithm.

The above chemometrics strategy, namely, with wavelet to select variables, then with PLS to compress data and reduce colinearity, and finally with BP-ANN to build quantitative calibration models, has been implemented with Matlab program. Figure 2 demonstrates its prediction errors in terms of root mean standard error of prediction (RMSEP), relative root mean standard error of prediction (RRMSEP), and correlation coefficient (R) between the actual concentrations of the validation samples and the predicted concentrations of theirs. From the figure, one could find that our strategy has a satisfactory precision.

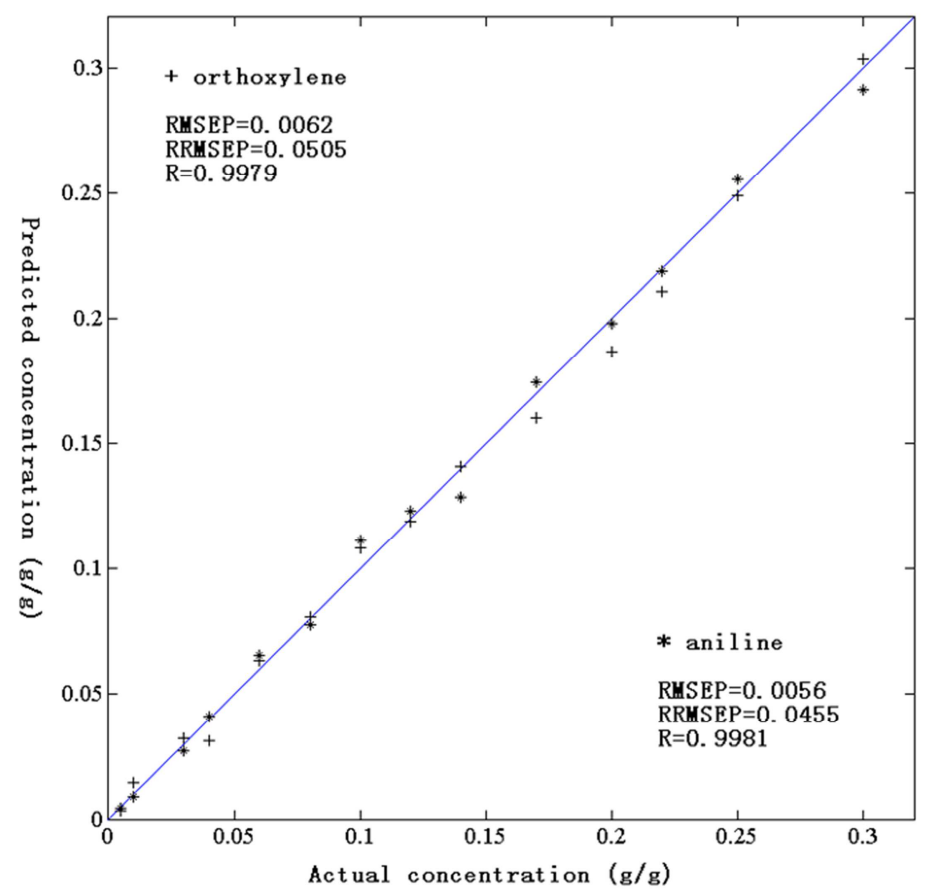

Figure 2. Correlation plots between the actual and the predicted concentrations of orthoxylene and aniline upon silica gel for validation samples.

\section{Results and Discussion}

With the NIR PCA experimental setup described in Section 2.2, spectra during the competitive adsorption process of orthoxylene/aniline vapor onto silica gel have been recorded and shown in Figure 3. It's worth noting that spectra shown in this figure were all collected with the silica gel adsorbent as a reference.

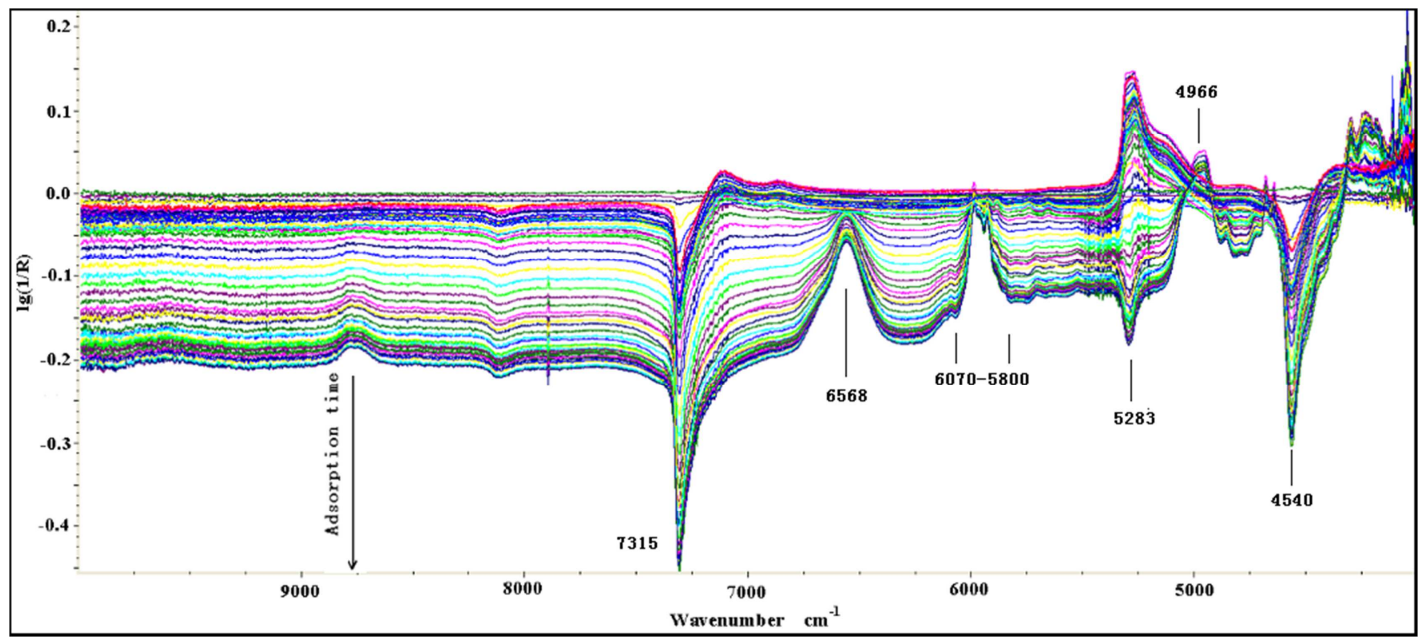

Figure 3. Raw NIR spectra during the competitive adsorption process of orthoxylenelaniline vapor onto silica gel (adsorption conditions were: temperature: $30^{\circ} \mathrm{C} ; \mathrm{P} / \mathrm{P}_{0}$ of orthoxylene: $0.50 ; \mathrm{P} / \mathrm{P}_{0}$ of aniline: $0.50 ; F_{0}: 400 \mathrm{~mL} / \mathrm{min}$; interval of recording time: $\left.1 \mathrm{~min}\right)$. 


\subsection{Equilibrium State of the Adsorption}

Based on our chemometrics strategy, the concentrations of each adsorbates at various adsorption times have been deduced from Figure 3 and demonstrated in Figure 4 (the left subplot).

It is very convenient for NIR PAC to determine whether the adsorption equilibrium state is reached (if the spectrum doesn't alter within five minutes). Form Figure 3 and 4, one

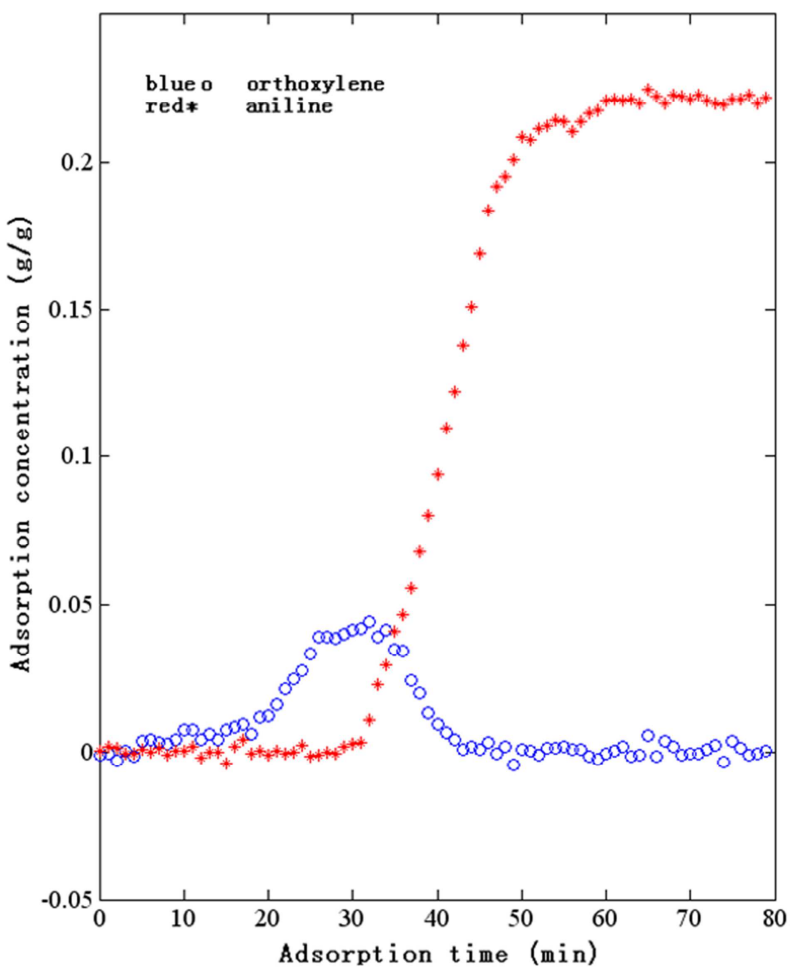

could discover that at about 62 minutes the adsorption achieved its equilibrium or stable state with aniline concentration of $0.22 \mathrm{~g} / \mathrm{g}$, and without any orthoxylene. The peak at $5283 \mathrm{~cm}^{-1}$ results from a small amount of water, which existed in the gas pipe and buffer jar of the experimental setup at the beginning of the adsorption process. Figure 3 shows that at the equilibrium state there was not any water adsorbed, too.

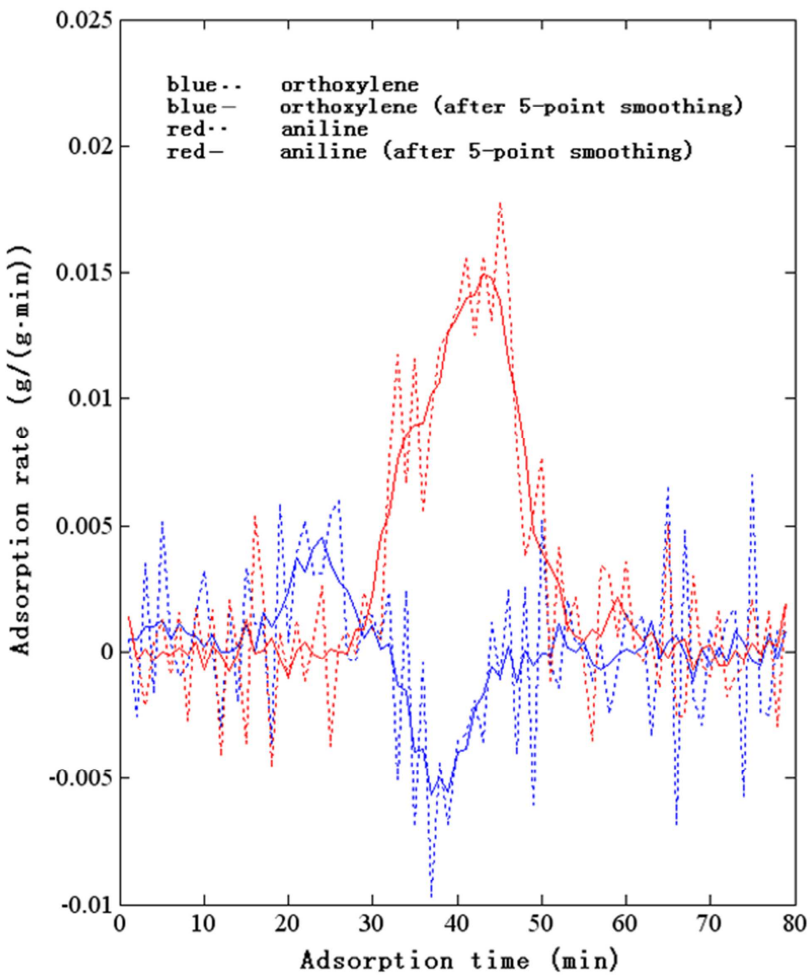

Figure 4. The adsorption concentrations of orthoxylene and aniline adsorbed by silica gel, and their adsorption rates during the adsorption process

\subsection{Adsorption Kinetics}

Since the adsorption bed (i.e., the quartz rectangle cell) is so small (length: $40 \mathrm{~mm}$; width: $10 \mathrm{~mm}$; thickness: $2 \mathrm{~mm}$ ) that it could be reasonably regarded as a differential adsorption bed [20], and because the NIR spectra were collected in a very short interval time (once every minute), the instantaneous adsorption rate could be nearly deduced from the difference between the adsorption amount at a time and that at the next time in terms of $\mathrm{g} /(\mathrm{g} \cdot \mathrm{min})$. The instantaneous adsorption rate during the adsorption process is also shown in Figure 3 (the right subplot). But due to analytical error these above instantaneous adsorption rates may not reflect the reality of the adsorption process, and consequently the smoothed adsorption rates are demonstrate in Figure 4 (the right subplot), too.

Figure 4 demonstrates that orthoxylene was adsorbed at first with an increasing adsorption rate until 24 minute, while at the same period aniline was not adsorbed at all. Then, the adsorption rate of orthoxylene decreased greatly to negative value (desorption) till the adsorbed orthoxyleen was all removed at 47 minute. As for aniline, at 29 minute silica gel began to adsorb it with a more and more rapid adsorption rate till 43 minute, and from 44 to 61 minute the adsorption rate decreased obviously till the equilibrium state of the adsorption process.

The fact that orthoxylene was adsorbed early but replaced by aniline finally can be attributed to their different adsorption states (see Section 4.3 for details): physical adsorption of orthoxylene resulted in more rapid adsorption rate but hydrogen-bonded adsorption of aniline was more stable.

\subsection{Adsorption State During the Competitive Adsorption Process}

For comparing spectral difference between before and after adsorption, NIR spectrum of orthoxylene (dissolved in $\mathrm{CCl}_{4}$ ), aniline (dissolved in $\mathrm{CCl}_{4}$ ), silica gel (before adsorption), orthoxylene (adsorbed upon silica gel), and aniline (adsorbed upon silica gel) have been recorded in Figure 5. According to reference [21], the absorbance wavebands or peaks of these spectra could be assigned as Table 1. 


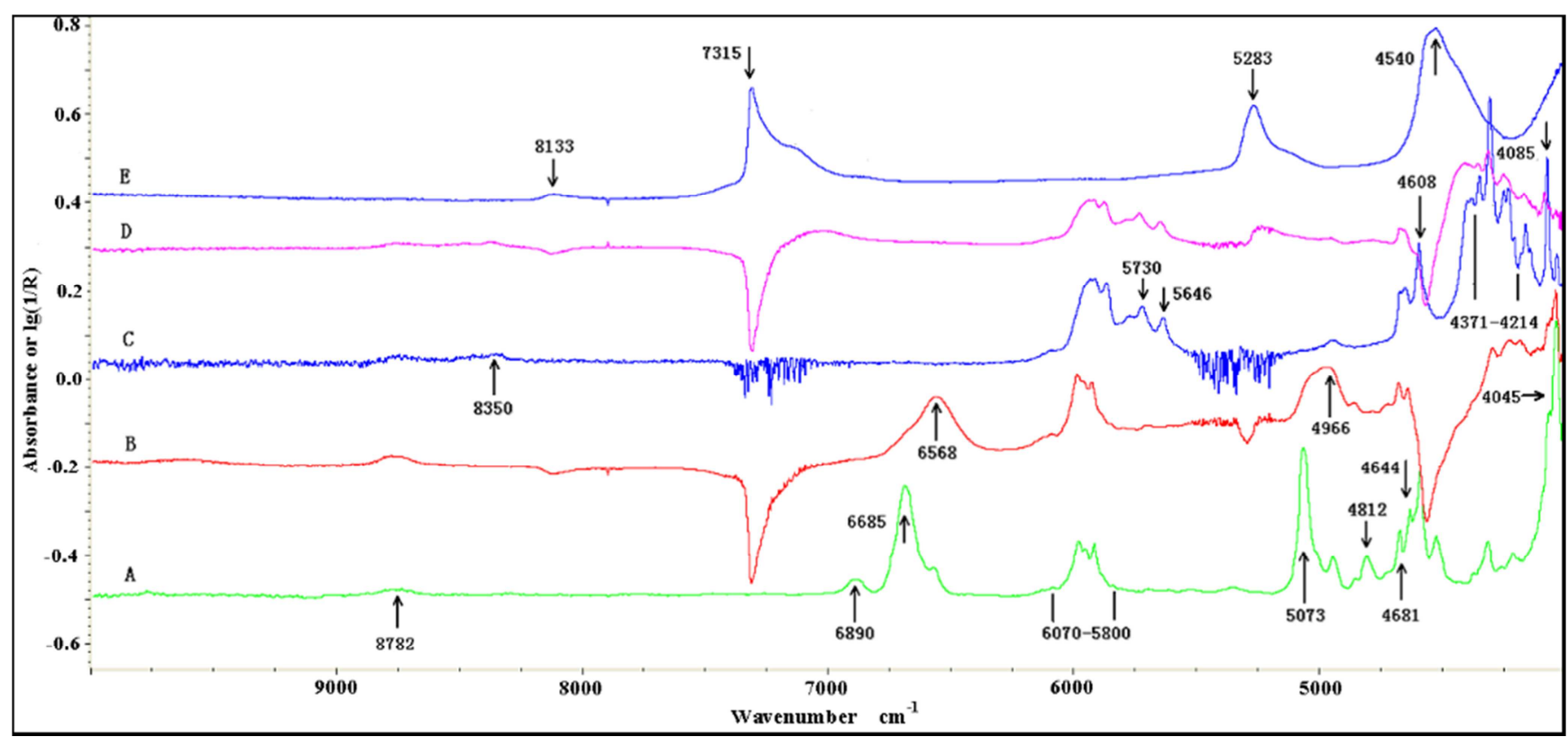

Figure 5. Raw NIR spectra of aniline in $\mathrm{CCl}_{4}$ solution ( $A, C C l_{4}$ as reference, detected in terms of absorbance), aniline absorbed by silica gel (B, silica gel as reference, detected in terms of $\lg (1 / R))$, orthoxylene in $\mathrm{CCl}_{4}$ solution $\left(C, C C l_{4}\right.$, absorbance), orthoxylene absorbed by silica gel (D, silica gel, $\left.\lg (1 / R)\right)$, silica gel before adsorption ( $E$, the reference accessory of the spectrometer, $\lg (1 / R)$

Table 1. The assignment of main NIR absorbance peaks and wavebands in Figure 2 and 4.

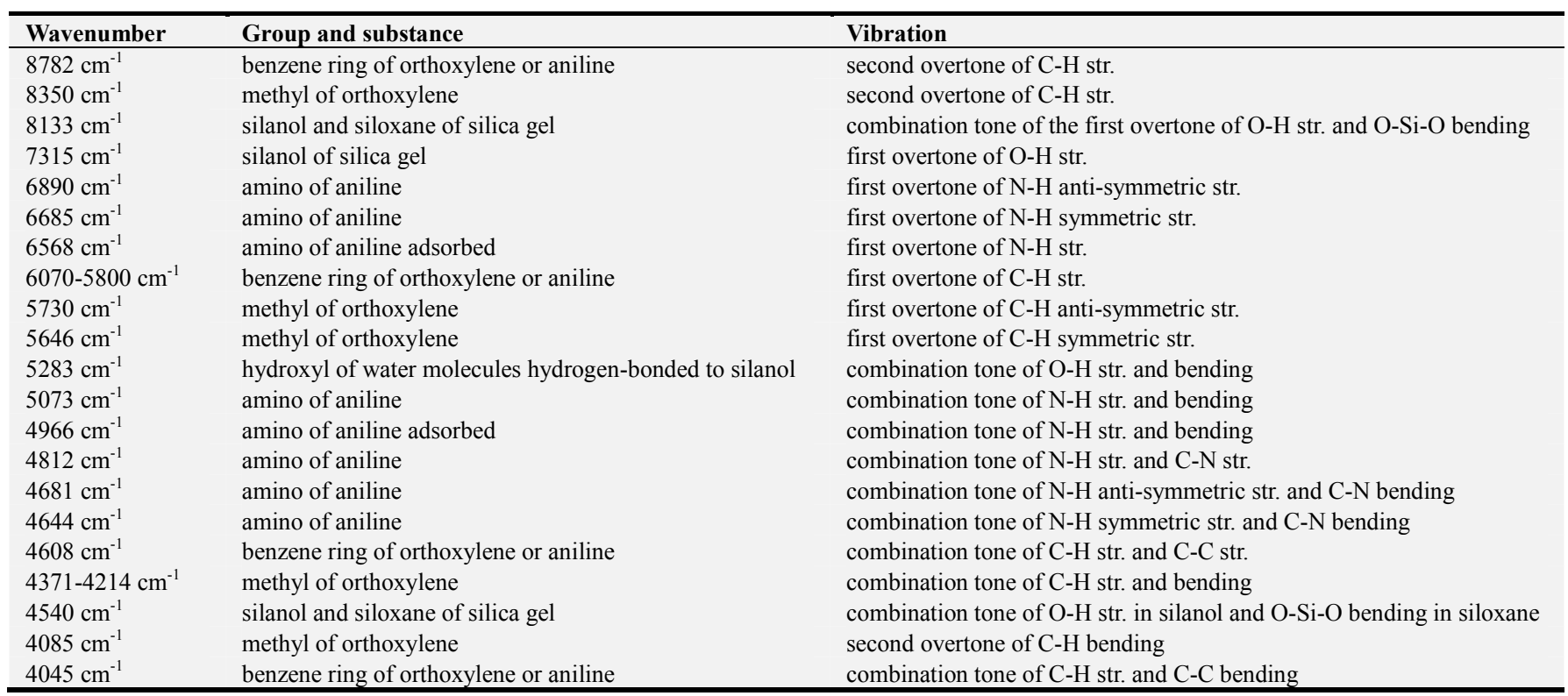

In Figure 3, and the spectrum $\mathrm{C}$ and D of Figure 5, all NIR peaks or wavebands of orthoxylene remained almost the same after it has been adsorbed by silica gel, indicating that the adsorption between it and silica gel was mainly physical.

According to Figure 3, and the spectrum A and B of Figure 5 , peaks merely related to alkyl and benzene ring also did not alter while those due to N-H vibration had typical red-shift (to longer wavelength and wider peak), such as 6685 to $6568 \mathrm{~cm}^{-1}$, 5073 to $4966 \mathrm{~cm}^{-1}$, suggesting that the adsorption was resulted from the formation of hydrogen bonds between aniline's amino groups and silanol groups on the silica gel surface.

This red-shift phenomenon continuing during the whole adsorption process means that all aniline molecules was adsorbed directly upon the surface of silica gel rather than upon orthoxylene or other aniline molecules, i.e., form a monolayer upon the surface of silica gel. This is also confirmed by synchronous kinetics data of Figure 4, in which the adsorption of aniline took place only if orthoxylene was desorbed during 29 to 44 minute. In addition, the spectrum of a sample containing $0.28 \mathrm{~g} / \mathrm{g}$ aniline (more than the concentration of adsorption equilibrium state) has also been collected, and is shown in Figure 6 with six spectra collected during various adsorption times. In the figure, only the spectrum of the sample has the peak at $6685 \mathrm{~cm}^{-1}$, and wavelet analysis of decomposition and reconstruction has separated these raw spectra into two peaks (in the figure, $\mathrm{A} 0 \approx \mathrm{A} 7+\mathrm{D} 5$ 
+D6 + D7), making difference more between the spectrum and

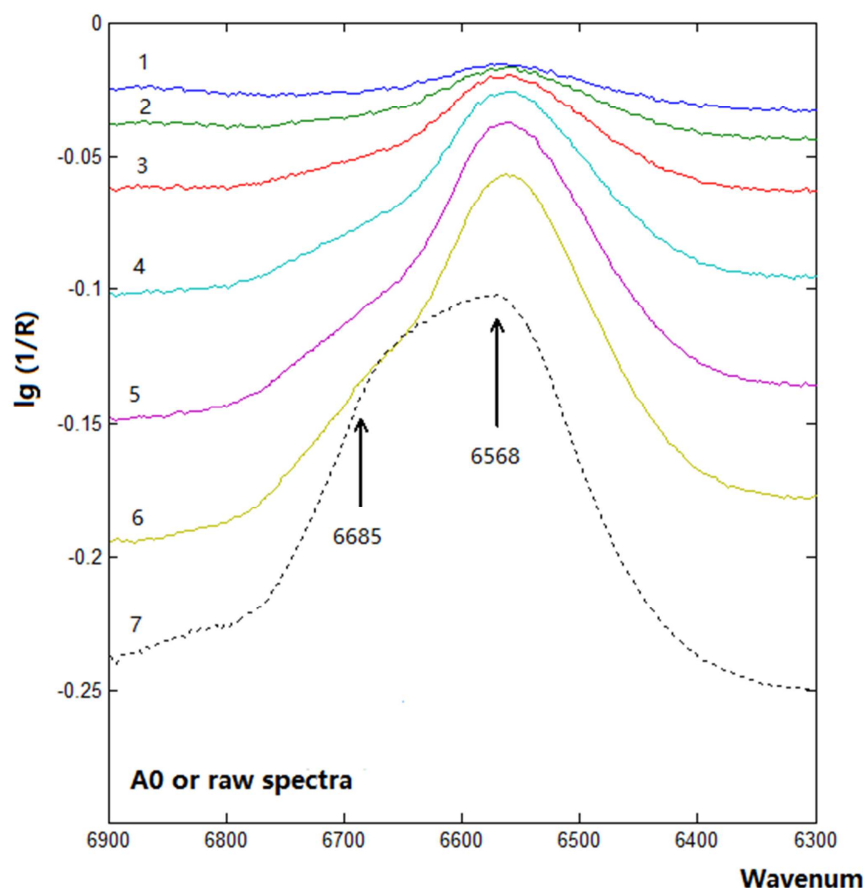

other six spectra more obvious.
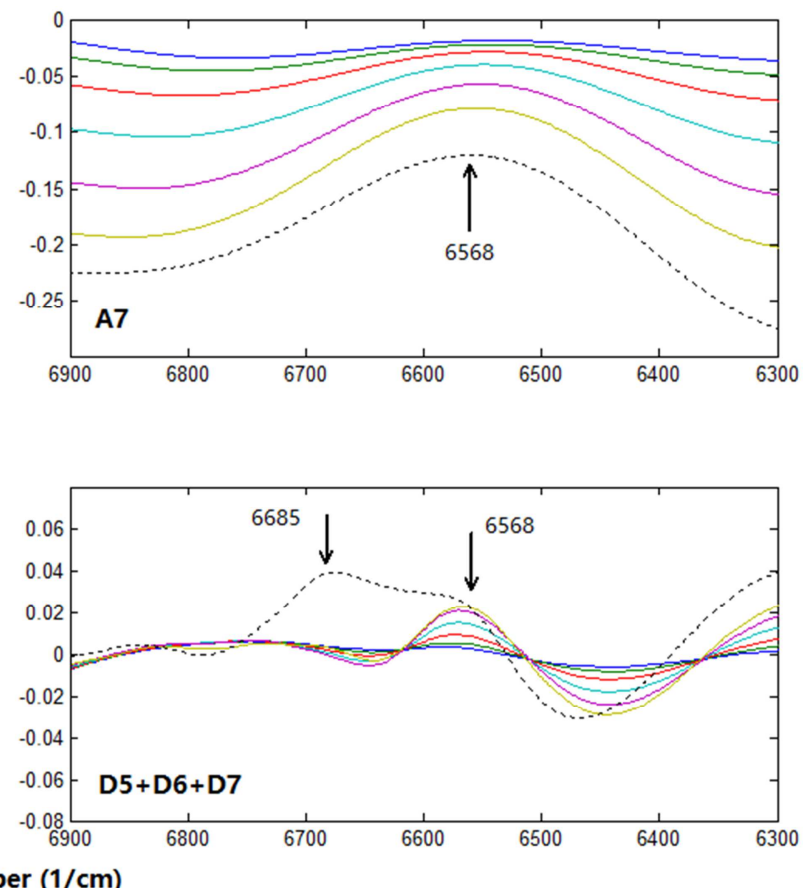

Figure 6. Raw NIR spectra of silica gel containing various aniline concentrations, and their reconstructed spectra after wavelet decomposition (Note: the adsorption time of spectrum 1, 2, 3, 4, 5, 6 is 33, 37, 45, 50, 55, 70 minute, respectively; the aniline concentration of spectrum 7 is 0.28 g/g).

The two negative peaks at 7315 and $4540 \mathrm{~cm}^{-1}$ of Figure 2 and 4 indicate the constraint of $\mathrm{O}-\mathrm{H}$ vibrations of silanol groups upon silica gel surface after it has adsorbed adsorbates. That is to say, when orthoxylene or aniline molecules have been adsorbed upon the adsorbent surface, the free vibration of O-H in silanol would be obstructed, resulting in two negative absorbance peaks (Note: spectra in Figure 3 were all collected with the silica gel before adsorption as the reference) [22]. As more and more adsorbate molecules had been adsorbed upon the silica gel surface, the two peaks became more and more negative.

The absorbance peak at $5283 \mathrm{~cm}^{-1}$ is due to water molecules hydrogen-bonded to silanol. Its strength increased at the beginning of adsorption process, indicating the adsorption of water, but soon decreased even to negative value, meaning the desorption of adsorbed water and even water contained in silica gel before the adsorption process.

\subsection{Further Information About the Competitive Adsorption Process}

Figure 4 also shows that at first aniline was not adsorbed upon empty surface of silica gel, but replaced orthoxylene or water molecules already adsorbed. The fact suggests that the surface of silica gel was not equal for adsorption, the places that have already adsorbed orthoxylene or water was more active to adsorb aniline, too.

As discussed above, the adsorption of aniline is due to the formation of hydrogen bonds between aniline's amino groups and silica gel's silanol groups. Therefore, it is reasonable to imagine that aniline was adsorbed uprightly, i.e., its benzene ring was vertical to the silica gel surface. For orthoxylene, it is difficult to determine how it was adsorbed on the surface of adsorbent merely by spectra. However, the fact that desorption rate of orthoxylene was roughly a third of the adsorption rate of aniline at the same time in Figure 4 may give a clue to the question. The area of aniline is nearly $0.23 \mathrm{~nm}^{2}$ when it is adsorbed uprightly upon the silica gel surface, while an orthoxylene molecule occupies roughly $0.67 \mathrm{~nm}^{2}$ if it lies on the silica gel surface horizontally. Therefore, it is reasonable to imagine an orthoxylene molecule was probably adsorbed with its benzene ring parallel to the surface of silica gel.

\section{Conclusion}

Through NIR PAC, a gas-solid competitive adsorption process of orthoxylene/aniline onto silica gel surface has been revealed thoroughly as well as clearly: orthoxylene and water was adsorbed at first, and then they were replaced promptly by aniline until the adsorption equilibrium state was reached at 61 minute with the aniline concentration of $0.22 \mathrm{~g} / \mathrm{g}$ and nearly without any orthoxylene or water; the adsorption of aniline was accompanied by the desorption of orthoxylene, and the adsorption rate is nearly three time of the desorption rate; the adsorption of aniline resulted from the hydrogen bonds between aniline's amino groups and silica gel's silanol groups while that of orthoxylene was due to physical adsorption; aniline was adsorbed vertically on the silica gel but orthoxylene laid evenly; some surface of silica gel was more active for adsorption than others. With the example, the NIR PAC methodology demonstrates its power to study multi-component or competitive gas-solid adsorption process. 


\section{Acknowledgements}

The authors are grateful to the financial support from the National Natural Science Foundation of China (Grant Nos. 21665002, 21665022).

$\mathrm{Lu} \mathrm{Xu}$ is also grateful to the financial support from Guizhou Provincial Science and Technology Department (No. QKHJC [2017] 1186), and the Talented Researcher Program from Guizhou Provincial Department of Education (QJHKYZ [2018] 073).

\section{References}

[1] Bartholdy, S.; Bjørner, M. G., Solbraa, E., Shapiro, A.; Kontogeorgis, G. M. Capabilities and limitations of predictive engineering theories for multicomponent adsorption. Industry \& Engineering Chemistry Research 2013, 52 (33), 1155211563

[2] Sircar, S. Basic research needs for design of adsorptive gas separation processes. Industry \& Engineering Chemistry Research 2006, 45 (16), 5435-5448.

[3] Sircar, S. Recent developments in macroscopic measurement of multicomponent gas adsorption equilibria, kinetics, and heats. Industrial \& Engineering Chemistry Research 2007, 46 (10), 2917-2927.

[4] Xomeritakis, G.; Tsai, C. Y.; Jeffrey, C. B. Microporous sol-gel derived aminosilicate membrane for enhanced carbon dioxide deparation. Seperation and Purification Technology 2005, 42 (2), 249-256.

[5] Brzić, D.; Petkovska, M. Some practical aspects of nonlinear frequency response method for investigation of adsorption equilibrium and kinetics. Chemical Engineering Science 2012, $82(12), 62-72$.

[6] Rynders, R. M.; Rao, M. B.; Sircar S. Isotope exchange technique for measurement of gas adsorption equilibria and kinetics. AIChE J. 1997, 43 (15), 2456-2463.

[7] Silverwood, I. P.; Keyworth, C. W.; Brown, N. J.; Shaffer, M. S. P.; Williams, C. K.; Hellgardt, K.; Kelsall, G. H.; Kazarian, S. G. An attenuated total reflection Fourier transform infrared (ATR FT-IR) spectroscopic study of gas adsorption on colloidal stearate-capped $\mathrm{ZnO}$ Catalyst Substrate. Applied Spectroscopy 2014, 68 (1), 88-94.

[8] Garrone, E.; Delgado, M. R.; Bonelli, B.; Arean, C. O. Probing gas adsorption in zeolites by variable-temperature IR Spectroscopy: an overview of current research. Molecules 2017, 22 (9) 1557-1565.

[9] Tumuluri, U.; Isenberg, M.; Tan, C. S.; Chuang, S. S. C. In situ infrared study of the effect of amine density on the nature of adsorbed $\mathrm{CO}_{2}$ on amine-functionalized solid sorbents. Langmuir 2014, 30 (25), 7405-7413.
[10] Workman, Jr. J.; Koch, M.; Veltkamp, D. J. Process analytical chemistry. Analytical Chemistry 2003, 75 (33), 2859-2882.

[11] Blanco, M.; Villarroya, I. NIR spectroscopy: A rapid-response analytical tool. Trends in Analytical Chemistry 2002, 21 (1), 412.

[12] Workman Jr., J.; Koch, M.; Veltkamp, D. J. Process analytical chemistry. Analytical Chemistry 2005, 77 (12), 3789-3806.

[13] Cai, C. B.; Han, Q. J.; Tang, L. J.; Zhang, Y.; Yu, R. Q. A novel method for studying multicomponent gas uptake on solid adsorbent with near-infrared process analytical technique. Industrial \& Engineering Chemistry Research 2008, 47 (18), $6835-6840$

[14] Cai, C. B.; Xu, L.; Zhong, W.; Tao, Y. Y.; Wang, B.; Yang, H. W.; Wen, M. Q. Studying a gas-solid multi-component adsorption process with near-infrared process analytical technique: Experimental setup, chemometrics, adsorption kinetics and mechanism. Chemometrics and Intelligent Laboratory Systems 2015, 144 (1), 80-86.

[15] Trygg, J.; Wold, S. PLS regression on wavelet compressed NIR spectra. Chemometrics and Intelligent Laboratory Systems 1998, 42 (1-2), 209-220.

[16] Cocchi, M.; Corbenllini, M.; Foca, G.; Lucisano, M.; Pagani. M. A.; Tassi, L.; Ulrici, A. Classification of bread wheat flours in different quality categories by a wavelet-based feature selection/classification algorithm on NIR spectra. Analytica Chimica Acta 2005, 544 (1-2), 100-107.

[17] Næs, T; Martens, H. Principal component regression in NIR analysis: Viewpoints, background details and selection of components. Journal of Chemometrics 1988, 2 (2), 155-167.

[18] Chen, Q.; Guo, Z.; Zhao, J.; Qin, O. Comparisons of different regressions tools in measurement of antioxidant activity in green tea using near infrared spectroscopy. Journal of Pharmaceutical and Biomedical Analysis 2012, 60 (1), 92-97.

[19] Marengo, E.; Bobba, M.; Robotti, E.; Lenti, M. Hydroxyl and acid number prediction in polyester resins by near infrared spectroscopy and artificial neural networks. Analytica Chimica Acta 2004, 511 (2), 313-322.

[20] Mayfield, P. L. J.; Do D. D. Measurement of the single-component adsorption kinetics of ethane, butane, and pentane onto activated carbon using a differential adsorption bed. Industry \& Engineering Chemistry Research 1991, 30 (3), $1262-1268$ 1262-1268.

[21] Workman, Jr. J.; Weyer, L. Practical guide to interpretive near-infrared Spectroscopy (Chinese edition). Chemical Industry Press: Beijing, 2009.

[22] Cai, C. B.; Han, Q. J.; Tang, L. J.; Xu, L.; Wu, H. L.; jiang, J. H.; $\mathrm{Yu}, \mathrm{R}$. Q. The spatial effect of near-infrared spectroscopy and its application to the study of supramolecular chemistry. Talanta 2009, 78 (2), 337-341. 